\title{
Field ion microscopy of nanoblocks on the surface of metals after irradiation argon ion beams
}

\author{
V. A. Ivchenko ${ }^{\dagger}$ \\ †ivchenko2008@mail.ru \\ Yeltsin Ural Federal University, 19 Mira str., Yekaterinburg, 620002, Russia \\ Institute of Electrophysics of Ural Branch of RAS, 106 Amundsena str., Yekaterinburg, 620016, Russia
}

\begin{abstract}
During irradiation of a platinum surface by beams of positive argon ions accelerated to an energy of $E=30 \mathrm{keV}$ up to fluence values of $F=10^{16}$ and $10^{17}$ ions $/ \mathrm{cm}^{2}$ at ion current densities of $150 \mu \mathrm{A} / \mathrm{cm}^{2}\left(T=70^{\circ} \mathrm{C}\right)$ and $200 \mu \mathrm{A} / \mathrm{cm}^{2}\left(T=200^{\circ} \mathrm{C}\right)$, respectively, an effect of nanoblocks formation has been detected. Basing of the results obtained by field ion microscopy method, an analysis of modification of the surface, subsurface volume and boundary areas of nanoblocks has been carried out. It is assumed that by their contrast, the boundary areas of nanoblocks correspond to the atomic structure of depleted zones. For a fluence of $F=10^{16} \mathrm{ion} / \mathrm{cm}^{2}$ nanoblocks were observed in a layer with an approximately $1.5 \mathrm{~nm}$ thickness under the irradiated surface. With an increase of the fluence up to $F=10^{17} \mathrm{ion} / \mathrm{cm}^{2}$, the effect of the of nanocrystalline block structure formation (with 1-5 nm sized blocks) is observed in a layer with the thickness not less than $20 \mathrm{~nm}$ under the irradiated surface. From the analysis of experimental data it follows that the mechanism of formation of such zones is likely to be related to the effect of channeling. The dimensions of the nanoblocks have been determined on the irradiated surface of Pt and in the subsurface volume as well. Histograms calculated from the experimental data give the size distribution of nanoblocks detected in the modified volume depending on the distance from irradiated surface. On the surface after irradiation with a fluence of $10^{16} \mathrm{ion} / \mathrm{cm}^{2}$, the fraction of blocks with an average size of $1 \mathrm{~nm}$ was $60 \%$. A change of the block size in the cross section of platinum sample with the distance from the irradiated (up to $10^{17} \mathrm{ion} / \mathrm{cm}^{2}$ ) surface has been revealed: with an increase of the latter up to $20 \mathrm{~nm}$ the average block size varies in the interval $1-5 \mathrm{~nm}$.
\end{abstract}

Keywords: interaction of ions with matter, structure modification, field ion microscopy.

УДК 538.9

\section{Полевая ионная микроскопия наноблоков на поверхности металлов после облучения пучками ионов аргона}

\author{
Ивченко В.А. ${ }^{\dagger}$ \\ Уральский федеральный университет им. первого президента России Б. Н. Ельцина, ул. Мира, 19, \\ Екатеринбург, 620002, Россия \\ Институт электрофизики Уральского отделения РАН, ул. Амундсена, 106, Екатеринбург, 620016, Россия
}

В результате облучения поверхности платины пучками ускоренных до энергии $E=30 \mathrm{keV}$ положительных ионов аргона до значений флюенса $F=10^{16}$ и $10^{17} \mathrm{ion} / \mathrm{cm}^{2}$ при плотностях ионного тока $150 \mu \mathrm{A} / \mathrm{cm}^{2}\left(T=70^{\circ} \mathrm{C}\right)$ и $200 \mu \mathrm{A} / \mathrm{cm}^{2}$ $\left(T=200^{\circ} \mathrm{C}\right)$ соответственно был обнаружен эффект образования наноблоков. На основании результатов, полученных методом полевой ионной микроскопии, проведен анализ модификации поверхности, приповерхностного объема и граничных районов наноблоков. Предполагается, что по контрасту граничные районы наноблоков соответствуют атомному строению обедненных зон. Для флюенса $F=10^{16} \mathrm{ion} / \mathrm{cm}^{2}$ наноблоки наблюдались в слое толщиной $\sim 1,5 \mathrm{~nm}$ под облученной поверхностью. При увеличении флюенса до $F=10^{17} \mathrm{ion} / \mathrm{cm}^{2}$ эффект формирования блочной нанокристаллической структуры (с размером блоков 1-5 nm) наблюдается в приповерхностном объеме толщиной не менее $20 \mathrm{~nm}$ от облученной поверхности. Из анализа экспериментальных данных следует, что, по-видимому, механизм образования таких зон связан с эффектом каналирования. Определены размеры наноблоков как на облученной поверхности Pt, так и в приповерхностном объеме. Гистограммы, рассчитанные из экспериментальных данных, показывают распределение обнаруженных в модифицированном объеме наноблоков по размерам в зависимости от расстояния от облученной. На поверхности после облучения с флюенсом $10^{16} \mathrm{ion} / \mathrm{cm}^{2}$, доля блоков со средним размером 1 нм составляла 60\%. Установлено изменение размеров блоков по сечению платинового образца с расстоянием от облученной (до $10^{17} \mathrm{ion} / \mathrm{cm}^{2}$ ) поверхности: с увеличением последнего до 20 nт средний размер блоков варьировался в пределах $1-5 \mathrm{~nm}$.

Ключевые слова: взаимодействие ионов с веществом, модификация структуры, полевая ионная микроскопия. 


\section{1. Введение}

Исследования изменений структуры металлов и сплавов после взаимодействия ускоренных заряженных пучков частиц с поверхностью показывают, что ионная обработка приводит к формированию особых конденсированных состояний, а также уникальных прочностных и физических свойств в материалах, которые не достигаются традиционными способами [1-3]. В результате развития этого направления радиационной физики твердого тела, возникают новейшие технологии, создаются новые материалы и покрытия.

В настоящей работе представлены данные экспериментального изучения строения наноблоков, возникающих в результате ионного облучения, и их граничных областей. Прямое исследование модификации поверхности структуры материалов, подвергнутых облучению ионами средних энергий $10-30 \mathrm{keV}$, экспериментально возможно с помощью метода полевой ионной микроскопии (ПИМ). ПИМ позволяет непосредственно визуализировать и регистрировать атомно-чистую поверхность, анализировать объект исследования в приповерхностном объеме с пространственным разрешением отдельных поверхностных атомов в процессе контролируемого удаления их атом за атомом, атомный слой за атомным слоем. Таким способом удается идентифицировать полученные на поверхности и в приповерхностном объеме структурные изменения вещества, возникшие под влиянием радиационного воздействия.

Цель настоящей работы - установить эффект образования нано структур на поверхности чистых металлах (на примере $\mathrm{Pt}$ ) после воздействия заряженных пучков ионов $\mathrm{Ar}^{+}$, и проанализировать изменения атомной поверхности и приповерхностного объема в зависимости от расстояния от облученной поверхности и флюенса.

\section{2. Техника эксперимента}

Объектом исследования служила поликристаллическая платина чистотой 99,99\%. Предназначенные для исследования образцы готовились в виде игольчатых эмиттеров с радиусом кривизны при вершине 30-50 nm из проволочных заготовок путем электрохимического полирования. Полевые эмиттеры после аттестации имели атомно-гладкую поверхность вершины острия, близкую к полусферической (Рис. 1а). Такую атомно-чистую поверхность получают in situ полевым испарением поверхностных атомов.
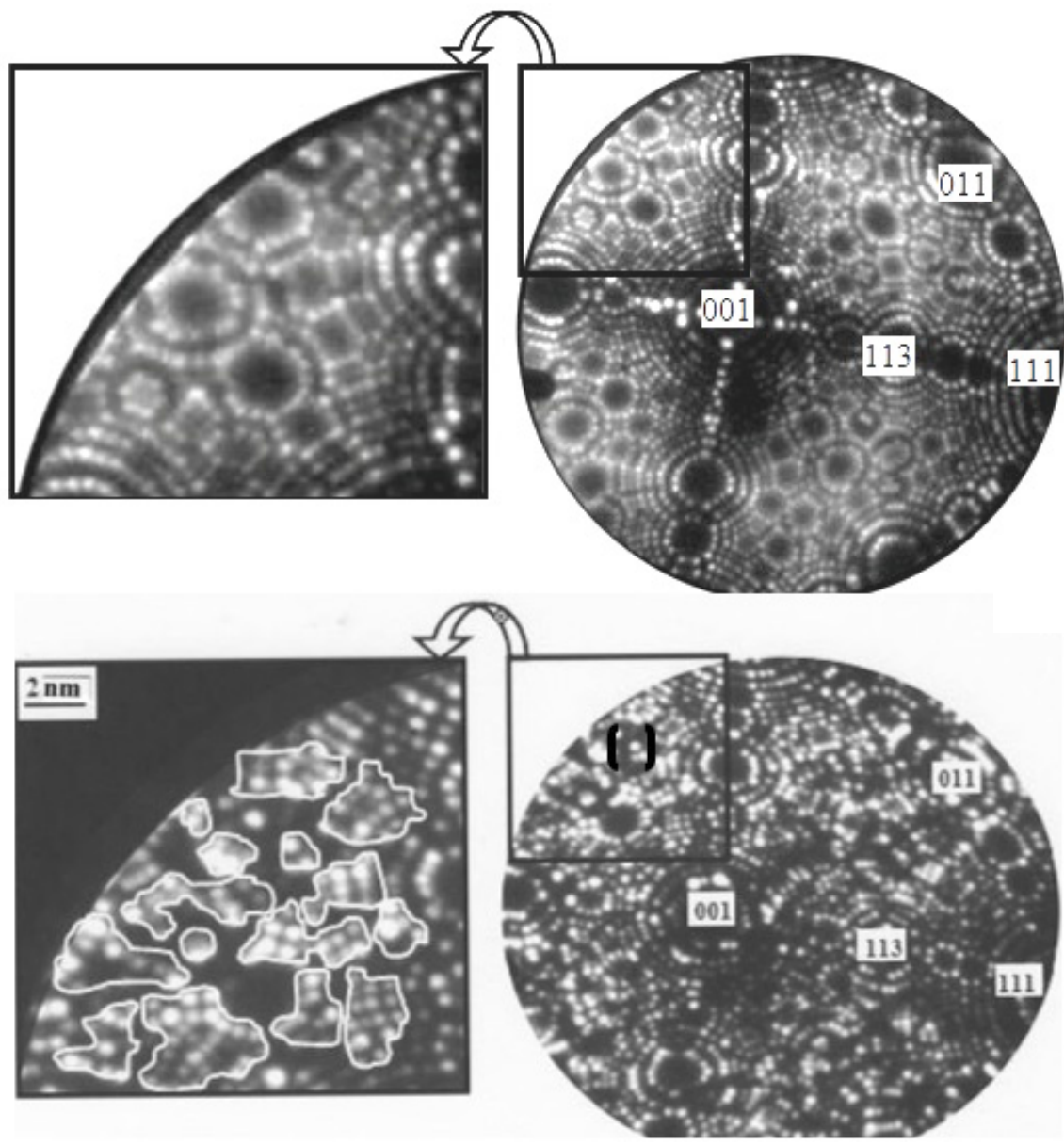

Рис. 1. Неоновые изображения одного и того же монокристалла Рt: аттестованный перед облучением кристалл (а); облученный ускоренными ионами аргона с $E=30 \mathrm{keV}, F=10^{16} \mathrm{ion} / \mathrm{cm}^{2}(\mathrm{~b})$. Контура показывают контраст наноблоков. Районы темного контраста между контурами предположительно указывают на обедненные зоны.

Fig. 1. Neon images of the same single crystal Pt: the crystal characterized prior to irradiation (a); irradiated with argon ions accelerated to $E=30 \mathrm{keV}, F=10^{16} \mathrm{ion} / \mathrm{cm}^{2}$ (b). Contours show the contrast of nanoblocks. Areas of dark contrast between the contours presumably indicate on depleted zones. 
Имплантированные после предварительной аттестации образцы-острия вновь помещали в ПИМ для изучения модифицированного объема сплава. Облучение предварительно аттестованных в полевом ионном микроскопе игольчатых платиновых образцов, проводилось пучками ускоренных до энергии $E=30 \mathrm{keV}$ положительных ионов аргона, флюенсом $F=10^{16}$ и $10^{17} \mathrm{ion} / \mathrm{cm}^{2}$ при плотностях ионного тока $150 \mu \mathrm{A} / \mathrm{cm}^{2}\left(T=70^{\circ} \mathrm{C}\right)$ и $200 \mu \mathrm{A} / \mathrm{cm}^{2}\left(T=200^{\circ} \mathrm{C}\right)$ соответственно.

Облученные образцы-острия вновь помещали в микроскоп и, регистрируя фото- или видеокамерой полевые ионные микрокартины поверхности при контролируемом удалении поверхностных атомов, получали экспериментальный материал для дальнейшего анализа поверхности и приповерхностного объема. Микрокартины поверхности регистрировались таким образом, чтобы количество испаренных атомов в промежутках между снимками было минимальным (в данной работе каждая микрофотография облученной поверхности регистрировались в процессе испарения полем одного атомного слоя грани (001), то есть по глубине на 0,2 nm). Такая процедура необходима для прецизионного выявления строения дефектов кристаллической решетки, возникших в результате взаимодействия с ускоренными пучками ионов $\mathrm{Ar}^{+}$.

Полевой ионный микроскоп был снабжен микроканальным ионно-электронным конвертером, усиливающим яркость ионных изображений в $10^{4}$ раз. Хладагентом служил жидкий азот $(T=78 \mathrm{~K})$, в качестве изображающего газа использовали спектрально чистый неон.

\section{3. Результаты и обсуждение}

После взаимодействия заряженных ионов аргона с металлом на микрокартинах атомно-чистой облученной поверхности (Рис. 1b) был зарегистрирован ионный контраст, отличный от ионной микрокартины чистого металла. Важно подчеркнуть, что такой контраст наблюдался на экране микроскопа после контролируемого удаления электрическим полем одного атомного слоя с грани (001) исследуемого образца Рt. Иными словами, напрямую регистрировалась облученная поверхность образца после флюенса $F=10^{16} \mathrm{ion} / \mathrm{cm}^{2}(E=30 \mathrm{keV})$, то есть с перекрываемыми каскадами атомных смещений. Анализ полученного ионного контраста облученной поверхности показал, что он кардинально отличается от ионного контраста поверхности предварительно аттестованного кристалла, изображенного на Рис. 1а. В данном случае, изменения ионного контраста облученной ионами аргона платины, по сравнению с контрастом исходной аттестованной $\mathrm{Pt}$, свидетельствуют о присутствии не только блочной наноразмерной структуры в приповерхностном объеме материала, но и зон темного контраста между блоками.

Именно нарушения в кольцевой картине ионного контраста трактуются как те или иные дефекты кристаллической структуры вещества и определяют соответствующий дефект, возникший в материале после различных внешних воздействий.
В рассматриваемом случае изменение ионного контраста свидетельствует об образовании блочной (Рис. 1b) нанокристаллической структуры в приповерхностном объеме материала (с размером блоков $1-3 \mathrm{~nm}$ ).

Кроме ярко выраженного контраста наноблоков, в каждом из которых атомы практически располагаются в узлах кристаллической решетки, на микрокартине поверхности наблюдаются ограничивающие их районы темного контраста. Ионный контраст этих участков (Рис. 1b), скорее всего, соответствует ионному контрасту обедненных зон на поверхности. Ранее [4], методом ПИМ регистрировались обедненные области после облучения быстрыми нейтронами платины и контраст нанопор.

Контраст темных областей, обнаруженный в настоящей работе, совершенно не соответствует типичному контрасту нанопор, обнаруженных ранее в сплаве 50Pd30Cu20Ag, (см. Рис. 2).

Нанопоры диаметром 3-12 nm и высотой 4-25 nm имели эллипсоидальную форму и были зарегистрированы после взаимодействия ускоренных до $24 \mathrm{keV}$ $\left(F=10^{18} \mathrm{ion} / \mathrm{cm}^{2}, j=300-340 \mu \mathrm{A} / \mathrm{cm}^{2}\right)$ положительных ионов аргона с атомно-чистой поверхностью предварительно деформированного на 70\% и отожженного при $T=850^{\circ} \mathrm{C}$ в течение 1 ч (с последующим охлаждением в воду) сплава.

В процессе изучения кристаллической структуры платины, облученной до $F=10^{16}-10^{18} \mathrm{ion} / \mathrm{cm}^{2}$ с энергией $30 \mathrm{keV}$ и $j=200 \mu \mathrm{A} / \mathrm{cm}^{2}$, помимо формирования наноразмерных блоков, в приповерхностном объеме также наблюдали ионный контраст нанопор. Такой контраст появлялся при флюенсе $10^{17} \mathrm{ion} / \mathrm{cm}^{2}$.

Контраст нанопор в Pt обычно фиксировался сразу же на ионном изображении поверхности и наблюдался в процессе удаления нескольких поверхностных атомных слоев имплантированного металла (Рис. 3а). Ионный контраст вакансионных нанопор в момент полевого испарения последнего перед появлением нанопоры атомного слоя, регистрировался в виде изображения

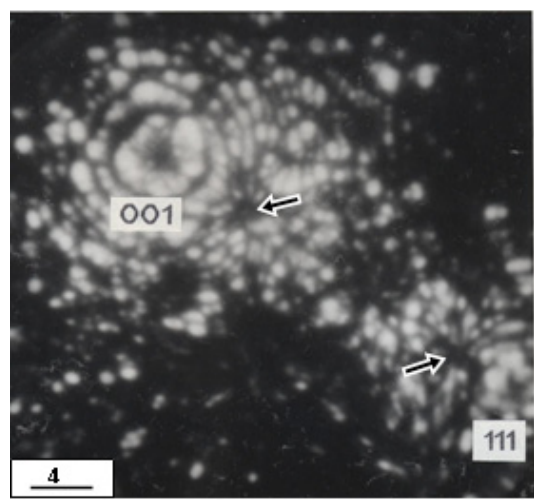

Рис. 2. Ионной контраст ионно-имплантированного сплава $50 \mathrm{Pd} 30 \mathrm{Cu} 20 \mathrm{Ag}$, после удаления 14 атомных слоев с облученной поверхности (стрелками отмечен ионный контраст нанопор) [4].

Fig. 2. An ion contrast from ion-implanted alloy 50Pd30Cu20Ag after removal of 14 atomic layers of the irradiated surface (arrows indicate ion contrast of nanopores) [4]. 
«кратеров». Затем, по мере полевого испарения атомных слоев, окружающих дефект, наблюдали сечение вакансионного кластера, размер которого был несколько меньше, чем при «вскрытии» нанопоры. И, наконец, «выход» нанопоры из материала при дальнейшем испарении атомных слоев с поверхности, как правило, заканчивался дислокационной петлей (Рис. 3b).

Отсюда с большой степенью вероятности можно говорить о наблюдении ограничивающих наноблоки областей как о прямом наблюдении обедненных зон на поверхности металла непосредственно после облучения заряженными пучками $\mathrm{Ar}^{+}$. Оценка ширины ограничивающих наноблоки участков из ионного контраста варьируется в диапазоне 1-5 нанометров. По размеру они соизмеримы с размерами наноблоков. По сути, наблюдаемая ионная микрокартина поверхности состоит из контраста обедненных зон и отдельных наноблоков.

В процессе последовательного контролируемого удаления поверхностных атомов электрическим полем было зарегистрировано достаточное количество микроснимков поверхности облученного металла, чтобы с большой степенью вероятности определить полученное модифицированное состояние приповерхностного объема. Анализируя ионный контраст облученной поверхности, можно с очевидностью утверждать, что ограничивающие наноблоки участки темного контраста, как правило, образуются в высокоиндексных гранях, то есть там, где ускоренным ионам $\mathrm{Ar}^{+}$энергетически выгоднее выбить поверхностные атомы из узлов кристаллической решетки металла. Темные области около кристаллографических наноблоков могут преимущественно испаряться за счет сильного смещения из узлов кристаллической решетки атомов материала. Мало смещенные из положений равновесия атомы, не испаряясь преимущественно, остаются видимыми, но имеют слабый контраст на фоне темного контраста, Рис. 4.

Предполагается, что центры областей темного контраста соответствуют центрам обедненных зон.

Изменения ионного контраста облученной $\left(F=10^{16} \mathrm{ion} / \mathrm{cm}^{2}\right)$ платины по сравнению с контрастом исходной аттестованной Pt наблюдались в слое толщиной $\sim 1,5 \mathrm{~nm}$ от облученной поверхности. Рис. 1b показывает, что атомы в наноблоках практически занимают свои узлы в кристаллической решетке материала, хотя сами наноблоки несколько разориентированы друг относительно друга. Принимая во внимание, что в настоящем эксперименте мишенью облучения служил монокристалл платины (объект исследования в микроскопе, имея радиус закругления $30-50 \mathrm{~nm}$, практически всегда является монокристаллом), можно предложить эффект каналирования [5,6] в качестве основного механизма образования такого модифицированного состояния при $F=10^{16}$ ion $/ \mathrm{cm}^{2}$. Проведенный количественный анализ размеров нанокристаллических блоков в зависимости от расстояния от облученной поверхности, обнаруженных в модифицированном приповерхностном объеме платины приводится на Рис. 5.

Для определения объемных долей наноблоков различных размеров использовался линейный метод А. Розиваля, основанный на принципе Кавальери-Акера.

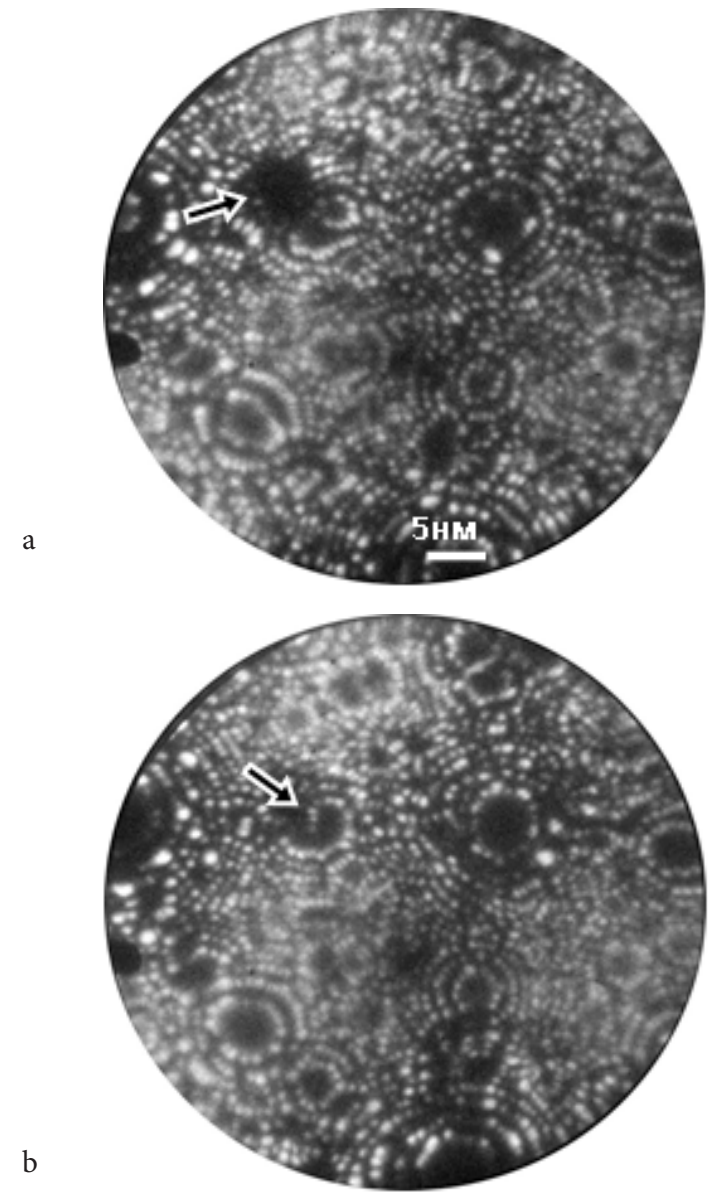

Рис. 3. Неоновые изображения чистой $\mathrm{Pt}$ после облучения ионами $\operatorname{Ar}^{+}\left(E=30 \mathrm{keV}, F=10^{18} \mathrm{ion} / \mathrm{cm}^{2}\right)$; типичный ионный контраст вакансионной нанопоры (показан стрелкой) (a); контраст дислокационной петли при выходе нанопоры из материала после полевого испарения 8 атомных слоев (001) (слоя толщиной $~ 1,6 \mathrm{~nm}$ ) (показан стрелкой) (b).

Fig. 3. Neon image of pure $\mathrm{Pt}$ after irradiation by $\mathrm{Ar}^{+}$ions $(E=30 \mathrm{keV}$, $\left.F=10^{18} \mathrm{ion} / \mathrm{cm}^{2}\right)$; typical ion contrast of a vacancy nanopore (indicated by an arrow) (a); contrast of a dislocation loop at an exit of a nanopore from the material after the field evaporation of 8 atomic (001) layers ( 1,6 $\mathrm{nm}$ thickness layer) (indicated by an arrow) (b).

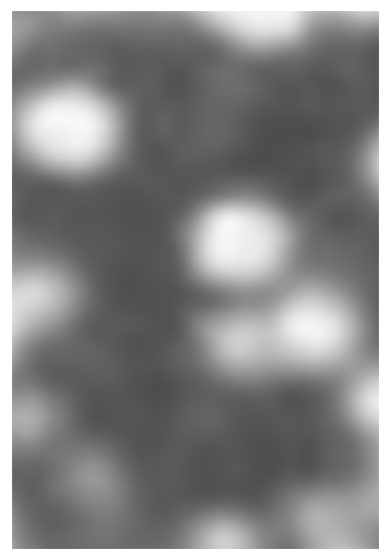

Pис. 4. Увеличенный участок ионного изображения облученной поверхности, соответствующий контрасту предполагаемой обедненной зоны, из рисунка $1 \mathrm{~b}$ (выделено черными скобками).

Fig. 4. An enlarged region of the ion image of the irradiated surface corresponding to the contrast of the presumably depleted area from figure $1 \mathrm{~b}$ (marked by black brackets). 
В результате анализа экспериментальных данных установлено, что после испарения полем приповерхностного объема толщиной 1,5 nm наблюдается контраст кольцевой микрокартины поверхности, практически соответствующей контрасту кольцевой картины необлученного кристалла, (Рис. 1a).

В процессе облучения $\mathrm{Pt}$ до более высокого флюенса $\left(F=10^{17} \mathrm{ion} / \mathrm{cm}^{2}\right)$, Рис. 6, эффект формирования блочной нанокристаллической структуры (с размером блоков 1-5 nm) наблюдается в приповерхностном объеме толщиной не менее $20 \mathrm{~nm}$ от облученной поверхности. Это демонстрирует Рис. 6, на котором приведена микрокартина участка поверхности исследуемого материала. В процессе контролируемого испарения полем поверхностных атомов, модифицированная пучками заряженных ионов аргона поверхность платины была изучена вплоть до 65-го атомного слоя от облученной поверхности, что соответствует удалению приповерхностного объема толщиной $20 \mathrm{~nm}$.

Анализ соответствующего экспериментального материала позволил измерить поперечные и продольные размеры нанокристаллических блоков и ширину граничной области между ними. Ширина граничной области, по нашим оценкам, варьировала от 0,8 до $2 \mathrm{~nm}$ на различных участках границ наноблоков в облученной ионами платине.

В $[7,8]$ было показано, что подобные нанокристаллические блочные структуры реализовывались в процессе интенсивных пластических деформаций. На основании этого можно предположить, что исследуемая в настоящей работе наноблочная структура после флюенса $\left(F=10^{17} \mathrm{ion} / \mathrm{cm}^{2}\right)$ является результатом деформационных процессов, протекающих в материале в ходе ионного облучения и в последующий за облучением промежуток времени.

\section{4. Заключение}

Получены прямые экспериментальные данные о расположении атомов на атомно чистой поверхности после облучения пучками ионов $\mathrm{Ar}^{+}$. В результате проведенных методом ПИМ исследований в ионно-имплантированной платине обнаружен эффект образования наноструктур как на поверхности, так и в приповерхностном объеме материала. Явление распространяется в объеме металла и зависит от флюенса $\left(F=10^{16}-10^{17} \mathrm{ion} / \mathrm{cm}^{2}\right)$ положительных ионов аргона при энергии $30 \mathrm{keV}$. Предполагается, что ограничивающие наноблоки районы, скорее всего, имеют атомное строение обедненных зон. Из анализа экспериментальных данных следует, что механизм образования таких зон связан с эффектом каналирования. Определены размеры наноблоков как на облученной поверхности Pt, так и в приповерхностном объеме материала. Рассчитанные из экспериментальных данных гистограммы показывают распределение обнаруженных наноблоков по размерам от расстояния от облученной поверхности в модифицированном объеме. На облученной поверхности при флюенсе $10^{16} \mathrm{ion} / \mathrm{cm}^{2}$, доля блоков со средним размером $1 \mathrm{~nm}$ составляла $60 \%$. Установлено изменение размеров блоков по глубине сечения платинового образца от облученной (при дозе $10^{17} \mathrm{ion} / \mathrm{cm}^{2}$ ) поверхности. Средний размер блоков варьировался в пределах $1-5 \mathrm{~nm}$ с увеличением расстояния до $20 \mathrm{~nm}$ по глубине.

Можно полагать, что вследствие формирования нанокристаллической структуры в приповерхностном объеме облученного ионами материала могут существенным образом повышаться его механические свойства (износостойкость, коррозионная стойкость). Получение таких наноструктур может оказаться перспективным для создания высоких поверхностных свойств материалов.

Благодарность/Acknowledgements. Paбота вblполнена в рамках темы государственного задания №0389-2015-0025 (на 2015-2017 г2.) и частично поддержана грантом РФФИ №. 16-08-00615./This work was performed as part of State Task No. 0389-2015-0025 and it was supported in part by RFBR Grant No. 16-08-00615.

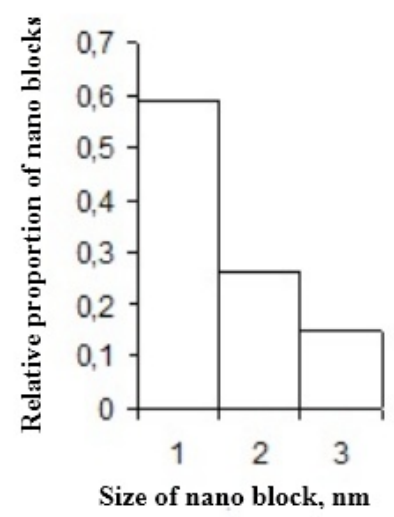

Рис. 5. Распределение блоков по размерам в модифицированном приповерхностном объеме платины, облученной ионами $\mathrm{Ar}^{+}$ $\left(E=30 \mathrm{keV}, F=10^{16} \mathrm{ion} / \mathrm{cm}^{2}\right)$.

Fig. 5. Size distribution of blocks in the modified subsurface volume of platinum irradiated by $\mathrm{Ar}^{+}$ions $\left(E=30 \mathrm{keV}, F=10^{16} \mathrm{ion} / \mathrm{cm}^{2}\right)$.

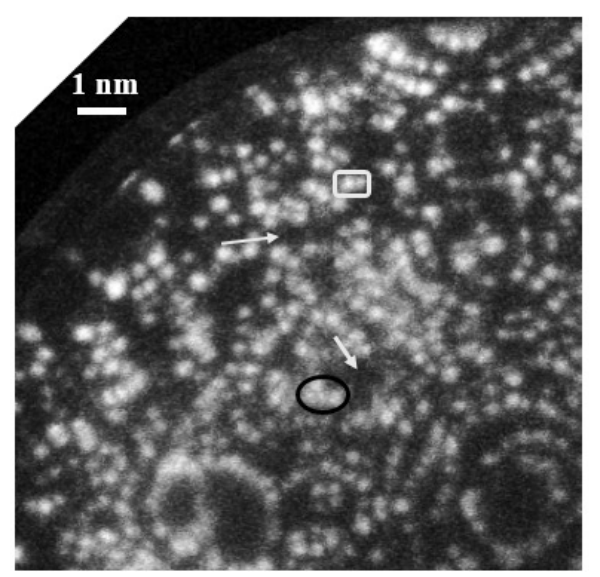

Рис. 6. Участок изображения поверхности платинового кристалла, облученного $\mathrm{Ar}^{+}\left(E=30 \mathrm{keV}, \quad F=10^{17} \mathrm{ion} / \mathrm{cm}^{2}\right)$; стрелками указаны обедненные зоны. Контура показывают контраст от типичных наноблоков.

Fig. 6. The image area of the surface of a platinum crystal irradiated by $\operatorname{Ar}^{+}\left(E=30 \mathrm{keV}, F=10^{17} \mathrm{ion} / \mathrm{cm}^{2}\right)$; arrows indicate depleted zone. Contours show the contrast from the typical nanoblocks. 


\section{Литература/References}

1. Surface Modification and Alloying by Laser, Ion, and Electron Beams, ed. by J.M. Poate, G. Foti, and D. Jacobson, M.: mechanical engineering (1987) 424. (in Russian) [Модифицирование и легирование поверхности лазерными, ионными и электронными пучками/Под ред. Дж. Поута, Г. Фоти и Д. Джекобсона, М.: Машиностроение (1987) 424].

2. V. V. Ovchinnikov. Izvestiay RAN. Metalli. 6. 104 (1996). (in Russian) [B.В. Овчинников. Известия РАН. Металлы. 6. 104 (1996)].

3. V.A. Ivchenko. Research of nanostructure states of materials after intensive external influences by atom probe FIM methods. 9th International Symposium of Croatian Metallurgical Society* S H M D '2010. Šibenik, June $20-24,212$ (2010).

4. V.A. Ivchenko, E. V. Medvedeva, V.V. Ovchinnikov. Poverkhnost, Rentgenovskie, Sinkhrotronnye i
Neitronnye Issledovaniya. 8. 26 (2009). (in Russian) [В.А. Ивченко, Е.В. Медведева, В.В. Овчинников. Поверхность. Рентгеновские, синхротронные и нейтронные исследования, 8, 26 (2009)].

5. M. Nastasi, J. W. Mayer, J. K. Hirvonen. Ion-Solid Interactions: Fundamentals and Applications.

Cambridge: Cambridge Solid State Science Series, Cambridge University Press XXVII. (1996) 540.

6. V.A. Ivchenko. Technical physics letters. 40. Is., 4. 323 - 325 (2014). DOI:10.1134/S1063785014040208

7. V.A. Ivchenko, B.M. Efros, E. V. Popova, N.B. Efros, L. V. Loladze. Physics and technique of high pressures. 13 (3). 109 - (2003). (in Russian) [В. А. Ивченко, Б. М. Эфрос, Е. В. Попова, Н. Б. Эфрос, Л. В. Лоладзе. Физика и техника высоких давлений. 13 (3). 109 (2003)].

8. B. M. Efros, E. V. Popova, N. B Efros, V.A. Ivchenko, V.N. Varyukhin. Metals. 6. 31 (2005). (in Russian) [Б. М. Эфрос, Е. В Попова, Н. Б. Эфрос, В. А. Ивченко, В.Н. Варюхин. Металлы, 6.31 (2005)]. 